Europhysics Letters

PREPRINT

\title{
Density functional theory of freezing for soft interactions in two dimensions
}

\author{
S. van Teeffelen $(*)$, C. N. Likos, N. Hoffmann and H. Löwen \\ Institut für Theoretische Physik II, Heinrich-Heine-Universität Düsseldorf, \\ Universitätsstraße 1, D-40225 Düsseldorf, Germany
}

PACS. 64.10.th - General theory of equations of state and phase equilibria. PACS. 64.70.Dv - Solid-liquid transitions.

PACS. 82.70.Dd - Colloids.

\begin{abstract}
A density functional theory of two-dimensional freezing is presented for a soft interaction potential that scales as inverse cube of particle distance. This repulsive potential between parallel, induced dipoles is realized for paramagnetic colloids on an interface, which are additionally exposed to an external magnetic field. An extended modified weighted density approximation which includes correct triplet correlations in the liquid state is used. The theoretical prediction of the freezing transition is in good agreement with experimental and simulation data.
\end{abstract}

A microscopic theory of freezing and melting is a great challenge in statistical physics. There are two complementary approaches to the liquid-to-solid transition: first, classical density functional theory [1-3] starts from liquid state and views the solid as a condensation of liquid density modes, hence it is a liquid-based approach. Second, crystal elasticity theory [4] is a solid-based theory where the liquid is viewed as a solid with an accumulation of defects. In three dimensions, the freezing transition is first order and it is known that it is not defect mediated. Here, density functional theory provides a molecular theory for the freezing transition. Crystal elasticity theory is appropriate to two dimensions and predicts a possible scenario of two-stage melting via an intermediate hexatic phase [5-8]. The advantage of density functional theory is that it can be used to calculate the structure of the solid, whereas it is not possible to extract the structure of the fluid out of crystal elasticity theory.

An excellent realization of a two-dimensional system is provided by paramagnetic colloidal particles in a pendant water droplet, which are confined to the air-water interface [9]. If an external magnetic field is applied perpendicular to the interface, a magnetic moment is induced in the particles resulting into a tunable, mutual dipolar repulsion between them. The corresponding interaction pair potential $u(r)$ is repulsive and soft, being proportional to $1 / r^{3}$, with $r$ denoting the distance between the particles. The prefactor can easily be tuned by varying the external magnetic field strength. In real-space experiments $[10,11]$, the twostage melting process was confirmed with an intermediate hexatic phase which had a tiny stability range bracketed between the fluid and crystalline phase. There are also computer

$\left({ }^{*}\right)$ E-mail: teeffelen@thphy.uni-duesseldorf.de

(c) EDP Sciences 
simulations $[12,13]$ for freezing in $1 / r^{3}$ system but finite-size effects turn out to become crucial here [14].

In this letter, we apply density functional theory (DFT) to two-dimensional freezing of soft $1 / r^{3}$ interactions. There are two major difficulties arising in doing so: first, it is known that it is difficult to get a reliable density functional approximation for soft repulsive interaction potentials. While hard sphere freezing serves as standard test case for various approximations and many reliable approximations do exist (e.g. Rosenfeld's fundamental measure theory [15]), the freezing of soft inverse-power law-fluids turns out to be much harder [16]. For the extreme case of the one-component plasma, featuring a $1 / r$ interaction, it has been shown by Likos and Ashcroft [17] that an extended modified weighted density approximation (EMA) which contains correct triplet correlation of the fluid yields reliable freezing data. In this letter, we overcome the first problem in a similar way and generalize the EMA to two dimensions and apply it to the $1 / r^{3}$ interaction. The second, more principal problem is linked to the twodimensional character of the system itself. It is not clear to date how to include the hexatic phase into the density functional language. Here, we do not address this deep question but rather focus on the prediction of the freezing transition point neglecting the tiny stability range of the hexatic phase. A similar view has been taken for hard disk systems [18-21] and to the $1 / r$ interaction in 2D [21] where density functional theory of freezing was applied to.

We demonstrate that the EMA yields excellent freezing data as compared to the standard modified weighted density approximation (MWDA) [22]. Even the relative-mean-squaredisplacement in the coexisting solid is in reasonable agreement with the experimental data on colloids in a magnetic field.

The Helmholtz free energy density functional is typically split into the ideal gas and an excess part, $F_{\text {tot }}[\rho(\boldsymbol{r})]=F_{\text {id }}[\rho(\boldsymbol{r})]+F_{\text {ex }}[\rho(\boldsymbol{r})]$. Here the ideal part is local and nonlinear, $F_{\text {id }}[\rho(\boldsymbol{r})]=k_{B} T \int \mathrm{d} \boldsymbol{r} \rho(\boldsymbol{r})\left\{\ln \left[\rho(\boldsymbol{r}) \Lambda^{3}\right]-1\right\}$, with $\Lambda$ denoting the thermal de Broglie wavelength and $k_{B} T$ the thermal energy. The excess part can only be calculated approximately. Both the MWDA and the EMA approximate the excess free energy of the inhomogeneous system by setting it equal to the excess free energy of a uniform liquid evaluated at an appropriately weighted density $\hat{\rho}$ :

$$
F_{\mathrm{ex}}[\rho(\boldsymbol{r})] \approx F_{\mathrm{ex}}^{\mathrm{MWDA} / \mathrm{EMA}}[\rho(\boldsymbol{r})]=N f_{0}(\hat{\rho}),
$$

where $N$ is the number of particles in the system and $f_{0}(\hat{\rho})$ is the excess free energy per particle of the uniform liquid at the weighted density

$$
\begin{aligned}
\hat{\rho}[\rho(\boldsymbol{r})]= & \frac{1}{N} \int \mathrm{d} \boldsymbol{r} \mathrm{d} \boldsymbol{r}^{\prime} \rho(\boldsymbol{r}) \rho\left(\boldsymbol{r}^{\prime}\right) w\left(\boldsymbol{r}-\boldsymbol{r}^{\prime} ; \hat{\rho}\right) \\
& +\frac{1}{N^{2}} \int \mathrm{d} \boldsymbol{r} \mathrm{d} \boldsymbol{r}^{\prime} \mathrm{d} \boldsymbol{r}^{\prime \prime} \rho(\boldsymbol{r}) \rho\left(\boldsymbol{r}^{\prime}\right) \rho\left(\boldsymbol{r}^{\prime \prime}\right) v\left(\boldsymbol{r}-\boldsymbol{r}^{\prime}, \boldsymbol{r}-\boldsymbol{r}^{\prime \prime} ; \hat{\rho}\right) .
\end{aligned}
$$

Here the second term only appears in the EMA and not in the MWDA. The weight functions $w(\boldsymbol{r} ; \rho)$ and $v\left(\boldsymbol{r}, \boldsymbol{r}^{\prime} ; \rho\right)$ are determined by requiring that in the uniform limit, $\rho(\boldsymbol{r}) \rightarrow \rho$ the approximate functional $F_{\text {ex }}[\rho(\boldsymbol{r})]$ is exact up to second (MWDA) or third (EMA) order in density difference $\Delta \rho(\boldsymbol{r})=\rho(\boldsymbol{r})-\rho$ in the functional expansion of the excess free energy of the inhomogeneous system about the excess free energy of the fluid. The second weight function $v$ in eq. (2) is chosen to be zero in the MWDA since the third order correlation function does not enter the formalism explicitly; rather, all higher terms are approximately included as a consequence of the self-consistency requirement on the determination of $\hat{\rho}$, appearing on both sides of eq. (2). The EMA, on the other hand, is exact up to third order and, similarly, includes 
approximate contributions from all higher-order terms. The normalized weight functions have to fulfill the requirements [17]

$$
\begin{aligned}
& \lim _{\rho(\boldsymbol{r}) \rightarrow \rho}\left[\frac{\delta^{2} F_{\mathrm{ex}}^{\mathrm{MWDA} / \mathrm{EMA}}}{\delta \rho(\boldsymbol{r}) \delta \rho\left(\boldsymbol{r}^{\prime}\right)}\right]=-k_{B} T c_{0}^{(2)}\left(\boldsymbol{r}-\boldsymbol{r}^{\prime} ; \rho\right) \\
& \lim _{\rho(\boldsymbol{r}) \rightarrow \rho}\left[\frac{\delta^{3} F_{\mathrm{ex}}^{\mathrm{EMA}}}{\delta \rho(\boldsymbol{r}) \delta \rho\left(\boldsymbol{r}^{\prime}\right) \delta \rho\left(\boldsymbol{r}^{\prime \prime}\right)}\right]=-k_{B} T c_{0}^{(3)}\left(\boldsymbol{r}-\boldsymbol{r}^{\prime}, \boldsymbol{r}-\boldsymbol{r}^{\prime \prime} ; \rho\right),
\end{aligned}
$$

where $c_{0}^{(2)}$ and $c_{0}^{(3)}$ are the two- and three-particle direct correlation functions of the liquid [23] which are an input to the theory. These conditions uniquely determine the weight functions and lead to simple algebraic equations for $v$ and $w$ that can be found in reference [17].

In order to find the equilibrium one-particle density $\rho_{\text {eq }}(\boldsymbol{r})$ we minimize the total free energy functional $F_{\text {tot }}^{\mathrm{MWDA} / \mathrm{EMA}}[\rho(\boldsymbol{r})]$ with respect to the inhomogeneous one-particle density $\rho(\boldsymbol{r})$. We make the Gaussian ansatz, $\rho(\boldsymbol{r})=\frac{\alpha}{\pi} \sum_{\boldsymbol{R}_{i}} \exp \left[-\alpha\left|\boldsymbol{r}-\boldsymbol{R}_{i}\right|^{2}\right]$ where $\left\{\boldsymbol{R}_{i}\right\}$ is the set of Bravais lattice vectors of a triangular lattice (with average density $\rho$ ). For fixed average density $\rho$ we thus end up with only one minimization parameter $\alpha$ which describes the degree of localization. For $\alpha \rightarrow 0$ the density profile becomes flat and the system turns into a homogeneous liquid of number density $\rho$, whereas increasing $\alpha$ leads to enhanced particle localization around the lattice sites.

With the Gaussian parametrization of the density profiles, we obtain the following selfconsistent equation for the weighted density $\hat{\rho}$ as function of the localization parameter $\alpha$ and the average density $\rho$ :

$$
\frac{\hat{\rho}(\rho, \alpha)}{\rho}=\left[1-\frac{k_{B} T}{2 f_{0}^{\prime}(\hat{\rho})} \sum_{\boldsymbol{K} \neq 0} \mu_{K}^{2} \tilde{c}_{0}^{(2)}(\boldsymbol{K} ; \hat{\rho})-\frac{\rho k_{B} T}{6 f_{0}^{\prime}(\hat{\rho})} \sum_{\substack{\boldsymbol{K} \neq \mathbf{0} \\ \boldsymbol{Q} \neq \mathbf{0},-\boldsymbol{K}}} \mu_{K} \mu_{Q} \mu_{|\boldsymbol{K}+\boldsymbol{Q}|} \tilde{c}_{0}^{(3)}(\boldsymbol{K}, \boldsymbol{Q} ; \hat{\rho})\right],
$$

where $\mu_{k}=e^{-k^{2} / 4 \alpha}$ are the Fourier coefficients of the Gaussian ansatz for $\rho(\boldsymbol{r})$ and, likewise, $\tilde{c}_{0}^{(n)}, n=2,3$, denote the Fourier transforms of the $n$-particle direct correlation functions, evaluated at the reciprocal lattice vectors (RLV's) $\boldsymbol{K}$ and $\boldsymbol{Q}$. Primes denote derivatives with respect to density and the three-particle term only appears in the EMA. Whereas $F_{\text {id }}$ grows with $\alpha, \hat{\rho}$ decreases with the latter, causing a concomitant decrease in $F_{\text {ex }}$, because the latter is a monotonically increasing function of $\hat{\rho}$. As can be induced from eq. (4) above, the decrease of $\hat{\rho}$ with $\alpha$ is pronounced when the RLV's of the lattice lie close to the maxima of $\tilde{c}_{0}^{(2)}(k ; \hat{\rho})$, a feature that corresponds physically to an inherent tendency of the fluid to enhance density waves at these wavevectors.

We now apply the MWDA/EMA to the inverse-power pair potential $u(r)=u_{0} / r^{3}$, where $u_{0}$ is a parameter with dimensions of energy $\times$ volume; for the specific realization of twodimensional paramagnetic colloids of susceptibility $\chi$ exposed to a perperndicular magnetic field $\mathbf{B}$, we have $u_{0}=(\chi \mathbf{B})^{2} / 2$. The thermodynamics and structure depend, due to simple scaling, only on one relevant dimensionless coupling parameter $\Gamma=u_{0} \rho^{3 / 2} /\left(k_{B} T\right)$. Therefore, it is convenient to express all quantities in terms of $\Gamma$ and consider coupling parameters rather than densities via this scaling relation. Correspondingly, in what follows we employ the weighted coupling constant $\hat{\Gamma}$, related to $\hat{\rho}$ via the scaling relation $\hat{\Gamma}(\Gamma, \alpha)=u_{0} \hat{\rho}^{3 / 2}(\rho, \alpha) /\left(k_{\mathrm{B}} T\right)$.

In order to obtain the concrete form of the functional approximations, we need the twoand three-particle direct correlation functions and the excess free energy per particle $f_{0}$ of the 


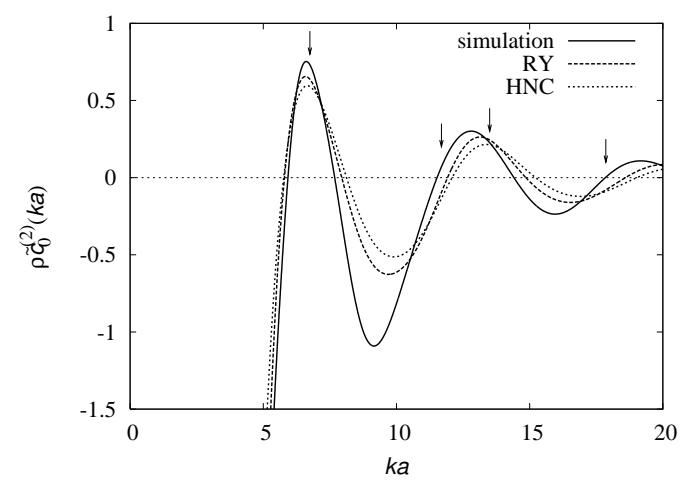

Fig. 1 - The Fourier transform $\rho \tilde{c}_{0}^{(2)}(k)$ of the two-particle direct correlation function at $\Gamma=9$, plotted against $k a$, where $a=\rho^{-1 / 2}$. Shown are simulation data using the Verlet closure (solid line); liquid integral equation theory using the RY closure (dashed line) and liquid integral equation theory using the HNC closure (dotted line). The arrows indicate the positions of the first four reciprocal lattice vectors of the triangular lattice.

corresponding uniform fluid for a wide range of coupling constants $\Gamma$. These quantities are obtained as described below:

(i) The two-particle direct correlation function is obtained by liquid integral equation theory, where we used the hypernetted chain (HNC) [23] or the thermodynamically consistent Rogers-Young (RY) closure [24]. We have also produced "exact" data for the real-space direct two-particle correlation by computer simulation using the Verlet closure [25]. A comparison between the HNC, RY and simulation data for the Fourier transform $\tilde{c}_{0}^{(2)}$ of the direct correlation function is shown in fig. 1 1 for the experimentally determined coupling close to freezing. The HNC closure underestimates the structure strongly while the RY closure is closer to the simulation data. We also show the positions of the first four reciprocal lattice vectors of a triangular lattice with same density. The value of $\tilde{c}_{0}^{(2)}$ at these lattice vectors crucially influences the solid free energies, as can be seen from eqs. (11) and (44). Therefore it can be anticipated that the $\mathrm{HNC}$ closure will not produce reliable results and we do not consider it further.

(ii) The excess free energy per particle $f_{0}$ is obtained from the pair correlation via the compressibility route.

(iii) Finally, the three-particle direct correlation function $c_{0}^{(3)}$ of the underlying fluid is obtained from an approximation by Denton and Ashcroft [26], which is based on a weighted density approximation to the first order direct correlation function of an inhomogeneous system. This approach leads to an analytic expression of $c_{0}^{(3)}$ in terms of the one- and two-particle correlation functions $c_{0}^{(1)}, c_{0}^{(2)}$ of the liquid and their derivatives with respect to density, employing the 'symmetrized sum'

$$
\tilde{c}_{0}^{(3)}\left(\boldsymbol{k}, \boldsymbol{k}^{\prime}\right)=\frac{1}{3}\left[\tilde{f}\left(|\boldsymbol{k}|,\left|\boldsymbol{k}^{\prime}\right|\right)+\tilde{f}\left(|\boldsymbol{k}|,\left|\boldsymbol{k}+\boldsymbol{k}^{\prime}\right|\right)+\tilde{f}\left(\left|\boldsymbol{k}^{\prime}\right|,\left|\boldsymbol{k}+\boldsymbol{k}^{\prime}\right|\right)\right],
$$

where

$$
\tilde{f}\left(k, k^{\prime}\right)=\frac{1}{c_{0}^{(1) \prime}}\left[\tilde{c}_{0}^{(2)}(k) \tilde{c}_{0}^{(2) \prime}\left(k^{\prime}\right)+\tilde{c}_{0}^{(2) \prime}(k) \tilde{c}_{0}^{(2)}\left(k^{\prime}\right)\right]-\frac{c_{0}^{(1) \prime \prime}}{\left[c_{0}^{(1) \prime}\right]^{2}} \tilde{c}_{0}^{(2)}(k) \tilde{c}_{0}^{(2)}\left(k^{\prime}\right) .
$$

Here, primes denote derivatives with respect to density. 


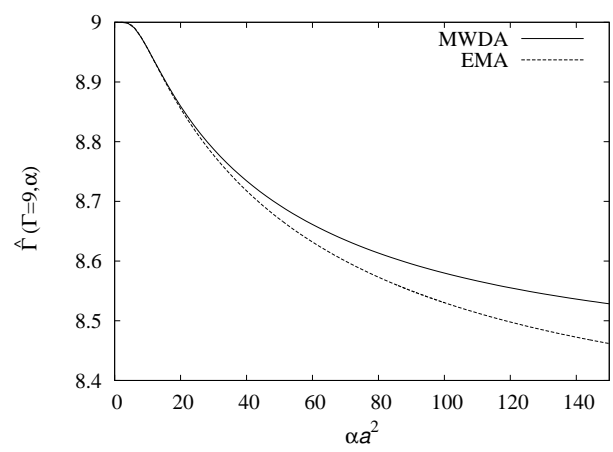

Fig. 2

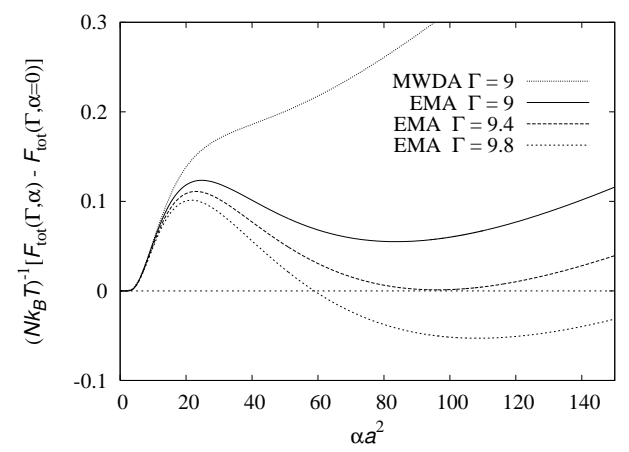

Fig. 3

Fig. 2 - The weighted coupling constant $\hat{\Gamma}(\Gamma, \alpha)$ as a function of the localization parameter $\alpha$ within the MWDA (solid line) and within the EMA (dashed line) using the "exact" pair structure from simulation for the strong coupling $\Gamma=9$.

Fig. 3 - Relative free energy per particle $N^{-1}\left[F_{\text {tot }}(\Gamma, \alpha)-F_{\text {tot }}(\Gamma, \alpha=0)\right]$ in units of $k_{B} T$ as a function of the localization parameter $\alpha$ obtained within the EMA for three different coupling constants $\Gamma=$ 9, 9.4, 9.8 (the three lower curves from top to bottom) compared to the relative free energy density obtained within the pure MWDA for a coupling constant $\Gamma=9$ (uppermost line).

Results for the approximations proposed are presented in figs. 2 and 3 In fig. 2 the weighted coupling constant $\hat{\Gamma}(\Gamma, \alpha)$ is shown versus the localization parameter $\alpha$ for a strong coupling $\Gamma$ close to freezing. Both the MWDA and the EMA are examined with the simulation pair structure input. Obviously, $\hat{\Gamma}$ coincides with the bare $\Gamma$ in the fluid $(\alpha=0)$. It can be seen that the MWDA yields a smaller reduction in $\hat{\Gamma}$ relative to $\Gamma$ than the EMA: explicit inclusion of three-body effects enhances the tendency of the particles to localize. Hence, one expects freezing at lower couplings in the EMA. In fact, in fig. 3 the free energy difference between a solid of localization $\alpha$ and a fluid $(\alpha=0)$ shown versus $\alpha$ reveals that the fluid is much more stable in the MWDA as compared to the EMA. The EMA yields a transition from the fluid to the solid close to $\Gamma=9.4$ : while for $\Gamma=9.0$ the fluid is stable as indicated by the minimal value at $\alpha=0$, fluid-solid coexistence is achieved at $\Gamma=9.4$, see the two equal minima in fig. 3 The solid phase, on the other hand, is clearly stable for $\Gamma=9.8$. The localization parameter at coexistence is roughly $\alpha_{\min } a^{2}=100$.

The full results of a numerical calculation using Maxwell's double tangent construction yield a weak first-order transition with a fluid density corresponding to a coupling constant of $\Gamma_{f}$ and a solid density corresponding to a coupling constant of $\Gamma_{s}$. There is a small density gap $\Delta \Gamma=\Gamma_{s}-\Gamma_{f}$, describing the coexistence region. Table I summarizes the freezing $/ \mathrm{melting}$ parameters for the MWDA with RY closure, for the EMA with RY closure, and for the EMA with the "exact" pair structure obtained from simulation. The data are compared against experimental results obtained from real-space microscopy measurement of magnetic colloids confined to an air-water interface. The experiments give freezing with an intermediate hexatic phase. The liquid-solid transition has also been studied using numerical simulation $[12,13]$ yielding a slightly higher inverse transition temperature between 12.0 and 12.25 but these investigations suffer from finite size effects.

As becomes evident from Table I, the MWDA is not a quantitatively satisfying theory as it overestimates the freezing coupling by a factor of 4 . Note that the overestimation of the freezing coupling is the reason why it is not possible to feed the "exact" pair structure 
TABLE I - Freezing and melting parameters $\Gamma_{f}$ and $\Gamma_{s}$, the widths of the coexistence regions $\Delta \Gamma=$ $\Gamma_{s}-\Gamma_{f}$, and the relative displacement parameters $\gamma$ at coexistence obtained within: the MWDA with the RY closure (first row); the EMA with the RY closure (second row); the EMA with the "exact" pair structure from simulation (third row) and experimental parameters for the isotropic-hexatic transition, the hexatic-crystal transition, and the Lindemann parameter, obtained from real-space microscopy measurements of magnetic colloids confined to an air-water interface (last row).

\begin{tabular}{l|llll} 
& $\Gamma_{f}$ & $\Gamma_{s}$ & $\Delta \Gamma$ & $\gamma$ \\
\hline MWDA with RY & 41.07 & 41.13 & 0.06 & 0.017 \\
EMA with RY & 23.0 & 23.08 & 0.09 & 0.020 \\
EMA with simulation & 9.33 & 9.49 & 0.16 & 0.020 \\
Experiment & 10.0 & 10.75 & - & 0.038
\end{tabular}

into the MWDA. At such high coupling, no fluid pair structures are available since the fluid spontaneously crystallizes in the simulation. The EMA, on the other hand, yields results in close agreement with experimental data.

More detailed, structural information can be extracted from the localization parameter of the coexisting solid. For all approximations used we find localization parameters at freezing in the range $99<\alpha_{\min }\left(\Gamma_{f}\right) a^{2}<115$. Strictly speaking, the localization parameter has no counterpart in "real" 2D systems since the particles are not localized due to long range fluctuations. However, if one relates the particle displacements to that of their nearest neighbor, one can define a finite quantity as $\gamma=\rho\left\langle\left(\boldsymbol{u}_{i}-\boldsymbol{u}_{i+1}\right)^{2}\right\rangle$, where $\boldsymbol{u}_{i}$ and $\boldsymbol{u}_{i+1}$ are the displacement vectors of neighboring lattice sites. Disregarding nearest-neighbor correlations $\left\langle\boldsymbol{u}_{i} \cdot \boldsymbol{u}_{i+1}\right\rangle$, $\gamma$ can be estimated. Since the nearest-neighbor correlations $\left\langle\boldsymbol{u}_{i} \cdot \boldsymbol{u}_{i+1}\right\rangle$ are expected to be positive:

$$
\gamma \lesssim 2 \rho\left\langle\boldsymbol{u}_{i}^{2}\right\rangle \approx 2 /\left(\alpha_{\min } a^{2}\right) .
$$

By this relation, the localization parameter of the coexisting solid gives a prediction for $\gamma$ which is included in Table I. From experiments, $\gamma$ is known to be close to $\cong 0.038$ [10]. This was shown to be in accordance with harmonic lattice theory [27]. The EMA yields $\gamma \lesssim 0.020$, i.e. the EMA roughly overestimates the localization of the particles by a factor of $2 . \gamma$ is smaller than the experimental value, contrarily to what was expected from the inequality (7). This shows that there is still a need to improve the theories in order to correctly predict localization properties. A similar overestimation of the localization is also common in weighted density approximations in three spatial dimensions [22].

In conclusion, we have demonstrated that the EMA is able to quantitatively predict the freezing transition of a two-dimensional colloidal system with soft and long-ranged $1 / r^{3}$ interactions in good agreement with experimental and simulation data. In analogy to threedimensional systems, the appearance of long-range interactions requires the explicit inclusion of three-particle correlation functions of the liquid in the construction of the weighted density. Furthermore, the predicted transition temperatures are very sensitive towards slight changes of the two-particle correlation functions of the underlying fluid. A highly accurate input of the same is therefore crucial.

Relying on the good quality of the EMA functional, our results can serve as a platform to treat more challenging problems than bulk transitions [28]. One obvious extension is towards external potentials acting on the particles, such as system walls or gravity. The MWDA can in principle be applied to a fluid near a single wall, but not to a free interface between coexisting phases. Another interesting example is a spatially inhomogeneous magnetic field, 
which renders the interactions space dependent [29]. Finally, one may employ the scheme of dynamical density functional theory $[30,31]$ in order to use the EMA functional to study the effect of spatially homogeneous magnetic fields that oscillate in time. Work along these lines is currently under way.

This work has been supported by the DFG within the SFB TR6, project section C3.

\section{REFERENCES}

[1] Oxtoby D. W. Nature, 347:725, 1990.

[2] Löwen H. Phys. Rep., 237:249, 1994.

[3] Singh Y. Phys. Rep., 207:351, 1991.

[4] Strandburg K. J. Rev. Mod. Phys., 60:161, 1988.

[5] Kosterlitz J. M. and Thouless D. J. J. Phys. C., 6:1181, 1973.

[6] Halperin B. I. and Nelson D. R. Phys. Rev. Lett., 41:121, 1978.

[7] Nelson D. R. and Halperin B. I. Phys. Rev. B, 19:2457, 1979.

[8] Young A. P. Phys. Rev. B, 19:1855, 1979.

[9] Zahn K., Méndez-Alcaraz J. M., and Maret G. Phys. Rev. Lett., 79:175, 1997.

[10] Zahn K., Lenke R., and Maret G. Phys. Rev. Lett., 82:2721, 1999.

[11] von Grünberg H. H., Keim P., Zahn K., and Maret G. Phys. Rev. Lett., 93:255703, 2004.

[12] Haghgooie R. and Doyle P. S. Phys. Rev. E, 72:011405, 2005.

[13] Löwen H. Phys. Rev. E, 53:R29, 1996.

[14] A critical survey of finite-size effects to hard disks can be found in Binder K., Sengupta S., and Nielaba P. J. Phys.: Condens. Matter, 14:2323, 2002; see also Bates M. A. and Frenkel D. Phys. Rev. E, 61:5223, 2000.

[15] Rosenfeld Y. Phys. Rev. Lett., 63:980, 1989.

[16] Wang D. C. and Gast A. P. J. Chem. Phys., 110:2522, 1999.

[17] Likos C. N. and Ashcroft N. W. Phys. Rev. Lett., 69:316, 1992; J. Chem. Phys., 99:9090, 1993.

[18] Zeng X. C. and Oxtoby D. W. J. Chem. Phys., 93:2692, 1990.

[19] Barrat J. L., Xu H., Hansen J. P., and Baus M. J. Phys. C., 21:3165, 1988.

[20] Xu H. and Baus M. J. Phys.: Condens. Matter, 2:5885, 1990.

[21] Ryzhov V. N. and Tareyeva E. E. Phys. Rev. B, 51:8789, 1995.

[22] Denton A. R. and Ashcroft N. W. Phys. Rev. A, 39:4701, 1989.

[23] Hansen J. P. and McDonald I. R. Theory of simple liquids. Academic Press, London, 2 edition, 1986.

[24] Rogers F. J. and Young D. A. Phys. Rev. A, 30:999, 1984.

[25] Verlet L. Phys. Rev., 165:201, 1968.

[26] Denton A. R. and Ashcroft N. W. Phys. Rev. A, 39:426, 1989.

[27] Froltsov V. A., Likos C. N., Löwen H., Eisenmann C., Gasser U., Keim P., and Maret G. Phys. Rev. E, 71:031404, 2005.

[28] Löwen H. J. Phys.: Condens. Matter, 13:R415, 2001.

[29] Froltsov V. A., Likos C. N., and Löwen H. J. Phys.: Condens. Matter, 16:S4103, 2004.

[30] Marconi U. M. B. and Tarazona P. J. Chem. Phys., 110:8032, 1999.

[31] Dzubiella J. and Likos C. N. J. Phys.: Condens. Matter, 15:L147, 2003. 